\title{
Exercise preserves muscle mass and force in a prostate cancer mouse model
}

\author{
Darpan I. Patel (1,2,3), Kira Abuchowski (1), Bilal Sheikh (1), Paul Rivas (6), Nicolas \\ Musi $(3,4,5)$, A. Pratap Kumar $(2,6)$
}

(1) School of Nursing, The University of Texas Health at San Antonio, San Antonio, TX, USA; (2) Mays Cancer Center at UT Health San Antonio MD Anderson Cancer Center, The University of Texas Health at San Antonio, San Antonio, TX, USA; (3) Barshop Institute for Longevity and Aging Studies, The University of Texas Health at San Antonio, San Antonio, TX, USA; (4) School of Medicine, The University of Texas Health at San Antonio, San Antonio, TX, USA; (5) The Geriatric Research Education and Clinical Center, South Texas Veterans Health Care System, San Antonio, TX, USA (6) Department of Urology, School of Medicine, The University of Texas Health at San Antonio, San Antonio, TX, USA

This article is distributed under the terms of the Creative Commons Attribution Noncommercial License (CC BY-NC 4.0) which permits any noncommercial use, distribution, and reproduction in any medium, provided the original author(s) and source are credited.

\begin{abstract}
The purpose of this study was to examine the effects of exercise in modulating biomarkers of sarcopenia in a treatment naïve transgenic adenocardinoma of the mouse prostate (TRAMP) model. Thirty TRAMP mice were randomized to either exercise (voluntary wheel running) or no-treatment control group for a period of 20 weeks. During necropsy, gastrocnemius muscles and prostate tumors were harvested and weighed. Gastrocnemius concentrations of myostatin, insulin-like growth factor (IGF)-1 and tumor necrosis factor (TNF)- $\alpha$ were quantified. Exercise mice had greater muscle mass than controls $(\mathrm{p}=0.04)$. Myostatin was significantly lower in the exercise group compared to controls $(\mathrm{p}=0.01)$. Exercise mice maintained forelimb grip force while control mice had a significaint decrease $(\mathrm{p}=0.01)$. No significant difference was observed in pre-post all limb grip strength. Further, forelimb and all limb grip strength was negatively associated with tumor mass $(\mathrm{p}<0.01)$.
\end{abstract}

Key Words: cachexia, sarcopenia, muscle strength, physical activity.

Eur J Transl Myol 29 (4): 328-333, 2019

Prostate cancer (PCa), an androgen dependent adenocarcinoma, is the second most common cancer among men. ${ }^{1}$ Treatment options vary including active surveillance, androgen deprivation therapy, chemotherapy, radiation therapy, and complete prostatectomy. While androgen deprivation therapy is considered the gold standard therapy for non-metastatic tumors, many patients are opting for active surveillance as a non-medical treatment option. No matter the treatment option planned, PCa patients are susceptible to muscle loss. ${ }^{2}$ The pathogenesis of sarcopenia involve a number of different factors. It is known that there are multiple biomarkers involved in the progression of sarcopenia, including loss of insulin-like growth factor (IGF)-1, and increased concentrations of myostatin and inflammatory cytokines. ${ }^{3}$ IGF-1, a stimulator of muscle growth, has been shown to be downregulated in patients with muscle loss. ${ }^{4}$ Conversely, increases in myostatin and the inflammatory cytokine tumor necrosis factor- alpha (TNF- $\alpha$ ) can overwhelm normal muscle physiology leading to atrophy and protein degradation. ${ }^{4}$ Moreover, myostatin gene expression is upregulated in the muscle of animals with tumor-induced muscle wasting. ${ }^{5}$ At this time, there are few therapies to manage sarcopenia. ${ }^{6}$ The most widely utilized therapies include pharmacological treatments, herbal medicines, exercise and dietary interventions., ${ }^{5,7}$ Recent studies have demonstrated that exercise is a viable option for patients with cancer, ${ }^{9}$ protecting against cancer associated muscle $\operatorname{loss}^{8}$ and promoting strength, endurance and quality of life. ${ }^{10}$ Animals models for $\mathrm{PCa}$ have shown to be effective and beneficial in aiding investigators to study the pathophysiology of $\mathrm{PCa}$ and to identify potential interventions to treat tumor progression and the secondary effects of cancer and cancer treatment. One of the popular animal models for the preclinical study of $\mathrm{PCa}$ is the transgenic adenomarcinoma of the mouse prostate (TRAMP) model. The TRAMP model is 
developed on the C57BL6 background and shares many of the characteristics of human PCa. ${ }^{11}$ In this model, tumor development mirrors clinical progression starting with low grade prostatic intraepithelial neoplasia (PIN) lesions that transition to high grade PIN lesions, well differentiated tumors to poorly differentiated tumors with metastasis. Thus, this tumor bearing mouse model can support foundational investigation on the effects of PCa on muscle physiology. The purpose of this study was to test the effects of exercise in modulating proteins associated with preserving muscle mass and force in a treatment naïve transgenic adenocarcinoma of mouse prostate (TRAMP) model. We reasoned that exercise can modify intramuscular concentrations of proteins related to sarcopenia on TRAMP mice while preserving muscle force similar to baseline.

\section{Materials and Methods}

\section{Ethics Statement}

This study was approved by the University of Texas Health at San Antonio Institutional Animal Care and Use Committee.

\section{Experimental design}

A randomized controlled study was used to test the research hypothesis. Thirty, 8-10 week old TRAMP mice obtained from Jackson Laboratories (Bar Harbor, ME) were used in the study and equally randomized to either the exercise group or a no-treatment control group for an intervention period of 20 weeks.

\section{Transgenic Mouse Model}

Prostate tumor development and progression in this model is akin to human prostate pathogenesis biochemically, pathologically and molecularly. ${ }^{12}$ TRAMP mice are developed on a pure C57BL/6 background by prostate-specific expression of SV40 large $\mathrm{T}$ antigen using the rat probasin promoter. Mice were housed individually in a temperature maintained environment with food and water available ad libitum. A 12 hour light/dark cycle was maintained. Body weight and food consumption were recorded weekly.

\section{Voluntary wheel running}

A $15.5 \mathrm{~cm}$ diameter low-profile running wheel, designed by Med Associates, Inc. (Georgia, VT), was placed in each cage of the exercising mouse group and was accessible at all times. The revolutions were recorded minute-by-minute using an electronic sensor that was imbedded into the wheel. This modality was chosen for the exercise intervention, as opposed to forced exercise modalities (i.e. forced treadmill running) because it is most reflective of natural mouse locomotion and behavior. ${ }^{13}$ Voluntary wheel running is characterized by short periods of performance at high speeds against a low load throughout the entire dark cycle. ${ }^{13}$ Additionally, forced exercise has the potential to induce a stress response that may alter the outcomes of our study. ${ }^{14,15}$

\section{Measuring Forelimb Muscle Force by Grip Strength Testing}

Skeletal muscle force production in mice was tested at baseline and prior to sacrifice using the Columbus Instruments Grip Strength Meter . Each mouse completed 10 repetitions on the apparatus with the first five repetitions using only the forelimbs and the last five repititions using all four limbs. Briefly, each mouse was gently lifted onto the stage and made to grab the pullgauge by holding them at the base of the tail. The gauge was pulled horizontally at a constate rate until the mouse released the gauge. Five sequential trials for the forelimbs was performed with a 1 minute rest between trials to avoid fatigue. The process was repeated for alllimb assessment. Forelimb and all limb grip strength was calculated using the associated computer software. Peak values and the average of the five trials was taken as the representative value for each animal. A fatigue index was caluuated as follows:

$\mathrm{FI}=\frac{\text { Peak force-lowest force }}{\text { peak force }} \times 100$

\section{Euthanasia and Tissue Collection}

All mice were euthanized with inhaled $\mathrm{CO}_{2}$ with exsanguination as a secondary method to confirm death. Bilateral gastrocnemius muscle was removed, rinsed clean, dried, weighed and snap frozen in liquid nitrogen until homogenization. Frozen samples were prepared for analysis by homogenizing a $1-2 \mathrm{~mm}$ piece of gastrocnemius muscle with $1 \mathrm{~mL}$ phosphate buffered saline using a glass Dounce homogenizer. Samples were then centrifuged at $1,500 \mathrm{~g}$ for five mins and supernatant was collected. At necropsy, the genitourinary (GU) complex, which includes prostate tumors, were also excised, cleaned, weighed and placed in $10 \%$ formalin for future histology assessments (histology data not presented).

\section{Gastrocnemius Protein Analysis}

Intramuscular concentrations of IGF-1 (ThermoScientific, Frederick, MD), myostatin (R\&D Systems, Minneapolis, MN), and TNF- $\alpha$ (ThermoFisher Scientific, Carlsbad, CA) were quantified in homogenized gastrocnemius muscle using commercially available assay kits following the manufacturer's recommended protocol. Briefly, gastrocneiums muscle was homogenized in phosphate buffered saline in volumes relative to the muscle mass using a dounce homogenizer. Supernatant was collected after centrifugation and used for protein analysis. Twenty microliters (myostatin) and $100 \mu \mathrm{l}$ (IGF-1 and TNF- $\alpha$, respectively) of supernatant were added to the respective plates for analysis. Sensitivity of each assay is as follows: IGF-1 (4 pg/ml), myostatin (5.32 pg/ml), TNF- $\alpha$ $(8 \mathrm{pg} / \mathrm{ml})$ All samples were assayed in duplicate and averaged. 
Table 1. Animal Characteristics

\begin{tabular}{lcc}
\hline & Control & Exercise \\
\cline { 2 - 3 } Body Mass (g) & $33.2 \pm 7.28$ & $35.2 \pm 8.5$ \\
Tumor Mass (g) & $4.92 \pm 7.79$ & $7.18 \pm 8.44$ \\
Tumor Free Body Mass (g) & $28.28 \pm 2.95$ & $28.02 \pm 2.13$ \\
Food Comsumption (g/week) & $26.06 \pm 3.57$ & $28.22 \pm 2.98^{*}$ \\
Running Activity (km/d) & --- & $13.56 \pm 4.1$ \\
Absolute Gastrocnemius Mass (g) & $0.41 \pm 0.16$ & $0.49 \pm 0.11^{*}$ \\
Relative Gasctrocnemius Mass (\%) & $1.37 \pm 0.4$ & $1.65 \pm 0.4^{*}$ \\
\hline
\end{tabular}

Data are presented as mean \pm standard deviation; *indicates significance between groups at p<0.05.
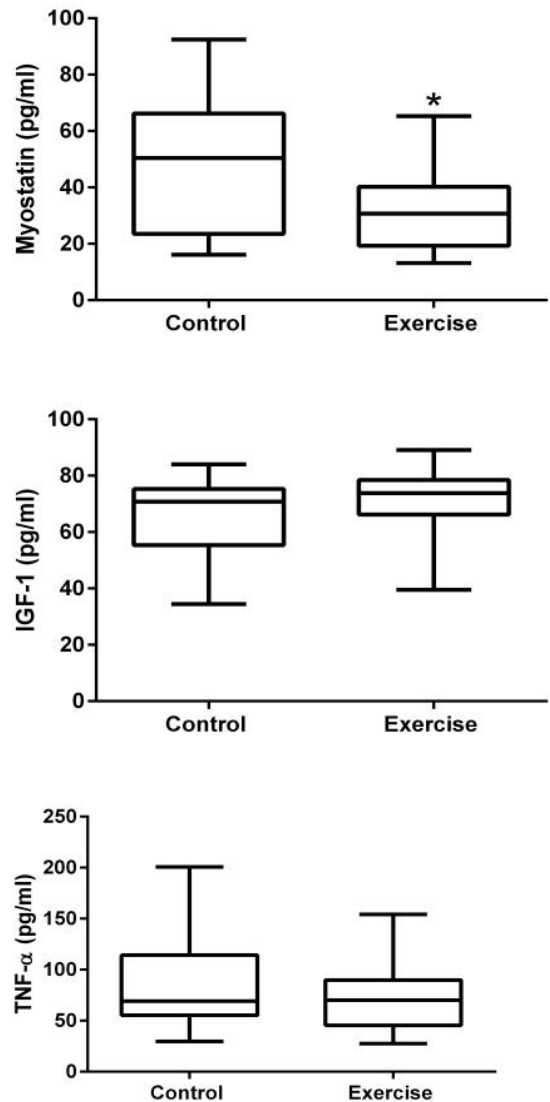

Fig 1. Intramuscular protein concentrations. Intramuscular concentrations of myostatin, IGF-1 and TNF- $\alpha$ were measured in homogenized gastrocnemius muscles. Exercise was found to have significantly lowered intramuscular myostatin concentrations $(p<0.05)$. Data are presented as $95 \%$ confidence interval.

\section{Statistical Analysis}

Based on previous studies conducted by our group, a sample size of 15 mice per group provides $80 \%$ power to detect significance changes between groups. Statistical analysis was completed using SPSS (IBM, Armonk, NY, USA) and presented in graphical form using GraphPad Prism (La Jolla, CA, USA). Student's t-test was performed to compare within and between group differences. Pearson's correlation analyses were performed to quantify relationships between tumor mass and outcome variables. Significance was set at $\mathrm{p}<0.05$ and data are presented as mean \pm standard deviation.

\section{Results}

The purpose of this study was to test the effects of exercise in modulating proteins associated with preserving muscle mass and force in treatment naïve TRAMP mice. No significant differences in tumor mass were observed with respect to treatment effect. Body mass increased in each of the groups across the 20 week intervention but no significant difference were found between groups. Tumor free body mass was also analyzed by taking the difference between total body mass and tumor mass. No significant treatment effect was observed across the 20 week study. Food consumption was significantly greater in the exercise group compared to the control group $(\mathrm{t}(27)=1.775, \mathrm{p}=0.04)$. Median running distance was not observed to change across the course of the experiment in the exercise group. Animal characteristic data is presented in Table 1.

\section{Exercise Increases Muscle Mass in TRAMP Mice}

Bilateral gastrocnemius mass was measured at the time of necropsy for all mice. The exercise group had significantly greater muscle mass compared to controls $(t(26)=2.333, p=0.01$; Figure 1$)$. In non-exercise control mice, tumor mass was negatively associated with gastrocnemius muscle mass $(\mathrm{r}=-0.53 ; \mathrm{p}=0.02)$. Exercise was observed to protect against tumor associated muscle loss $(\mathrm{r}=-0.355 ; \mathrm{p}>0.05)$. 
Table 2. Muscle Force Production

\begin{tabular}{|c|c|c|}
\hline & Control & Exercise \\
\hline \multicolumn{3}{|l|}{ Forelimb } \\
\hline Avg at Baseline & $96.36 \pm 11.89$ & $90.17 \pm 12.63$ \\
\hline Avg at End of Study & $79.86 \pm 10.35^{* * * *}$ & $88.87 \pm 13.99^{\wedge}$ \\
\hline Peak at Baseline & $126.7 \pm 19.90$ & $127.2 \pm 30.14$ \\
\hline Peak at End of Study & $106.2 \pm 18.85^{* *}$ & $115.5 \pm 18.03$ \\
\hline \multicolumn{3}{|l|}{ All Limb } \\
\hline Avg at Baseline & $206.6 \pm 25.40$ & $178.4 \pm 22.57$ \\
\hline Avg at End of Study & $199.1 \pm 38.68$ & $190.8 \pm 42.73$ \\
\hline Peak at Baseline & $252.3 \pm 31.32$ & $223.5 \pm 36.79$ \\
\hline Peak at End of Study & $262.5 \pm 80.63$ & $254.2 \pm 71.13$ \\
\hline \multicolumn{3}{|l|}{ Fatigue Index $(\%)$} \\
\hline Forelimb at Baseline & $46.37 \pm 9.31$ & $50.98 \pm 17.49$ \\
\hline Forelimb at End of Study & $50.44 \pm 15.50$ & $44.45 \pm 10.91$ \\
\hline All limb at Baseline & $34.72 \pm 7.78$ & $32.64 \pm 14.50$ \\
\hline All limb at End of Study & $34.48 \pm 17.34$ & $37.98 \pm 10.04$ \\
\hline \multicolumn{3}{|c|}{$\begin{array}{l}\text { Data are presented as mean } \pm \text { standard deviation; } \\
* * \text { indicates significance at } p<0.01 \text { within group }\end{array}$} \\
\hline
\end{tabular}

\section{Myostatin Significantly Reduced with Exercise}

Intramuscular myostatin concentrations suggest that exercise is capable of reducing intramuscular myostatin concentrations at greater rates than compared to controls. Specifically, exercise mice had significantly lower absolute $(\mathrm{t}(25)=2.141, \mathrm{p}=0 / 02)$ and relative $(\mathrm{t}(25)=2.754$, $\mathrm{p}=0.005)$ myostatin concentrations compared to controls. No significant differences in IGF-1 or TNF- $\alpha$ concentrations were observed with respect to timepoint nor treatment effect.

\section{Grip Force Preserved in Treatment Groups}

Within group comparisons revealed that control mice had significantly lower grip force in the forelimb at the end of the study compared to baseline $(\mathrm{t}(12)=3.66, \mathrm{p}=0.001$; Table 2). No significant differences were observed in exercise. Further, forelimb peak force was significantly lower at the completion of the study in the control group compared to baseline $(\mathrm{t}(12)=2.91, \mathrm{p}=0.01)$. At the end of the study, forelimb grip force was significantly greater in the exercise group $(\mathrm{t}(23)=1.840, \mathrm{p}=0.04)$. A negative association was observed in the control group between tumor mass and all limb grip strength $(r=-.820$, $\mathrm{p}=<0.001)$. The exercise group also demonstrated an association between tumor mass and forelimb grip strength $(r=-.833, p=<0.001)$ but not all limb grip strength $(r=-.417, p>0.05)$. No significant differences were observed in forelimb or all limb fatigue index rates within groups or between groups before or after the study.

\section{Discussion}

The maintenance in muscle mass and strength observed in the exercise group has a significant impact on quality of life in men with PCa.10, 16 The gastrocnemius muscle has greater recruitment of muscles fibers due in part to the greater metabolic activity of this muscle. 2 In this study, TRAMP mice that exercised had significantly greater muscle mass compared to sedentary controls. While the pathophysiology of sarcopenia and muscle wasting is complex, the combination of catabolic proteins, tumor derived factors, 8 changes in resting metabolism along with reduced protein synthesis, insulin sensitivity and increased protein breakdown are known to contribute to this catastrophic condition. ${ }^{17}$ Interestingly, the exercise group presented with a larger tumor mass than the control, leading us to hypothesize that exercise may play a role in hyperplasia or hypertrophy of prostate tissue. The negative association between tumor mass and grip force in the exercise group may be related to an increase in tumor derived factors that impact muscle morphology and function. Our group and othes have demondstrated that tumor derived proteins 
alter muscle mass in tumor challenged models. ${ }^{8,9}$ The results of this study lead us to hypothesize that these factors amy also play a role in muscle function as well. A potential explanation for this positive outcome is the reduced intramuscular concentrations of myostatin. Myostatin plays a significant role in initiating catabolic activity in skeletal muscle by negatively regulating Akt signaling and promoting SMAD $2 / 3$ signaling that increases protein degradation and muscle wasting through MuRF1 and MAFbx signaling. ${ }^{18}$ Reducing myostatin leads us to hypothesis that exercise in tumor bearing mice protects against muscle loss by decreasing myostatin signaling and thus preventing atrophy and promoting positive protein turnover. Future work by our group will focus on testing this hypothesis. Upwards of $60 \%$ of men with PCa develop musle loss regardless of treatment option. ${ }^{19}$ This project aims to model treatment naïve PCa that is synonymous with active surveillance in the clinical population. Esau and colleagues recently reported that $\mathrm{PCa}$ reduces exercise capacity in association with changes in muscle mass in an orthotopic animal model of the cancer. ${ }^{2}$ The effect of reduced exercise capacity in the clinical population has a tremendous effect on patients leading to increased fatigue status and reduced quality of life. The results of this study suggest that exercise is capable of preserving muscle contractile force as evident by the significant differences in muscle force generation at the end of the experiemtnal period. Additional outcome variables not measured provide limitations to the interpretation of the outcomes. First, exercise capacity, cage activity or spontaneous activity was not measured. The existence of the tumor, which varied in size, may have hindered activity in non-exercising mice, influencing one or more of our results. Use of a non-tumor control group would have provided more evidence to the benefits of exercise. Muscle cross sectional area would also provide value to the results of this study and should be included to explain muscle morphology. Lastly, downstream proteins of myostatin and activation of Akt signaling were not measured in our study and warrant detailed investigation. In conclusion, exercise is able to prevent muscle loss by decreasing myostatin concentrations in skeletal muscle. Successfully, both interventions were able to preserve muscle function. The present study has important implications for $\mathrm{PCa}$ patients that chose on active surveillance as a care plan. These outcomes of our study suggest that exercise is able to protect skeletal muscle from the deleterious effects of $\mathrm{PCa}$ with likely implications on muscle strength, endurance, fatigue and quality of life.

\section{List of acronyms}

TRAMP - transgenic adenocarcinoma of the mouse prostate

IGF - insulin-like growth factor

TNF - tumor necrosis factor

$\mathrm{PCa}$ - prostate cancer
PIN - prostatic intraepithelial neoplasia

FI - fatigue index

\section{Authors contributions}

DP, KA, PR, data collection; DP, KA,BS, NM, APK, data analysis, manuscript writing and revisions.

\section{Acknowledgments}

None.

\section{Funding}

The work was supported by National Cancer Institute designated UT Health San Antonio Cancer Center (P30 CA054174) Population Science and Prevention pilot grant (DP). DP is also supported by grants from the Department of Education (P031S150048) and from the NIH (U01 AR071150). NM is supported by the San Antonio Claude D. Pepper Older Americans Independence Center (P30 AG044271), the San Antonio Nathan Shock Center of Excellence on the Biology of Aging (P30 AG021890) and grants from the NIH (R01DK80157 and R01-DK089229), and the American Diabetes Association. APK is supported by the CTRC 40th Anniversary Distinguished Professor of Oncology Endowment and the National Center for Complementary and Alternate Medicine (1R01 AT007448).

\section{Conflict of Interest}

The authors have no conflicts to disclose.

\section{Ethical Publication Statement}

We confirm that we have read the Journal's position on issues involved in ethical publication and affirm that this report is consistent with those guidelines.

\section{Corresponding Author}

Darpan I. Patel, PhD, Associate Professor/Research, School of Nursing, University of Texas Health Science Center at San Antonio, 7703 Floyd Curl Drive, Mail Code 7975, San Antonio, Texas 78229, USA.

Tel. +1.210.567.0362 Fax. +1.210.567.1719

Email: pateld7@uthscsa.edu

E-mails of co-authors

Kira Abuchowski: kiraabuchowski@gmail.com

Bilal Sheikh: bsheikh24@gmail.com

Paul Rivas: rivasp@uthscsa.edu

Nicolas Musi:musi@uthscsa.edu

A.Pratap Kumar: kumara3@uthscsa.edu

\section{References}

1. Siegel RL, Miller KD, Jemal A. Cancer statistics, 2016. CA: a cancer journal for clinicians 2016;66:730.

2. Esau PJ, Gittemeier EM, Opoku-Acheampong AB, et al. Prostate cancer reduces endurance exercise capacity in association with reductions in cardiac and skeletal muscle mass in the rat. Am J Cancer Res 2017;7:2566. 


\section{Exercise and muscle properties in a mouse model of prostate cancer}

Eur J Transl Myol 29 (4): 328-333, 2019

3. Miyamoto Y, Hanna DL, Zhang W, et al. Molecular Pathways: Cachexia Signaling-A Targeted Approach to Cancer Treatment. Clin Cancer Res 2016;22:3999-4004.

4. Costelli P, Muscaritoli M, Bossola M, et al. IGF-1 is downregulated in experimental cancer cachexia. Am J Physiol-Reg I 2006;291:R674-83.

5. Zhou X, Wang JL, Lu J, et al. Reversal of cancer cachexia and muscle wasting by ActRIIB antagonism leads to prolonged survival. Cell 2010;142:531-43.

6. Fearon K. Cancer cachexia: developing multimodal therapy for a multidimensional problem. Eur $\mathbf{J}$ Cancer 2008;44:1124-32.

7. Tazi EM, Errihani H. Treatment of Cachexia in Oncology. Indian J Palliative Care 2010;16:129-37.

8. Patel DI, Wallace D, Abuchowski K, et al. Nexrutine ${ }^{\circledR}$ preserves muscle mass similar to exercise in prostate cancer mouse model. Physiol Rep 2019;7:e14217.

9. Lenk K, Schuler G, Adams V. Skeletal muscle wasting in cachexia and sarcopenia: molecular pathophysiology and impact of exercise training. J Cachexia, Sarcopenia and Muscle 2010;1:9-21.

10. Schmitz KH, Courneya KS, Matthews C, et al. American College of Sports Medicine roundtable on exercise guidelines for cancer survivors. Med Sci Sports Exerc 2010;42:1409-26.

11. Hurwitz AA, Foster BA, Allison JP, et al. The TRAMP mouse as a model for prostate cancer. Curr Protoc Immunol 2001; Chapter 20:Unit 20.5. doi: 10.1002/0471142735.im2005s45.

12. Kumar AP, Bhaskaran S, Ganapathy M, et al. Akt/CREB/Cyclin D1 network: a novel target for prostate cancer inhibition in transgenic adenocarcinoma of mouse prostate (TRAMP) model mediated by Nexrutine $\AA$, a Phellodendron amurense bark extract. Clin Cancer Res 2007;13:2784-94.

13. Novak CM, Burghardt PR, Levine JA. The use of a running wheel to measure activity in rodents: Relationship to energy balance, general activity, and reward. Neurosci Biobehav Rev 2012;36:100114.

14. Hoffman-Goetz L, Pedersen BK. Exercise and the immune system: a model of the stress response? Immunology Today 1994;15:382-7.

15. Patel D, White L, Lira V, Criswell D. Forced exercise increases muscle mass in EAE despite early onset of disability. Physiol Res 2016;65:101317. Epub 2016 Aug 19.

16. Galvao DA, Spry N, Denham J, et al. A Multicentre Year-long Randomised Controlled Trial of Exercise Training Targeting Physical Functioning in Men with Prostate Cancer Previously Treated with Androgen Suppression and Radiation from TROG 03.04 RADAR. Eur Urol 2014;65:856-64.

17. Bowen TS, Schuler G, Adams V. Skeletal muscle wasting in cachexia and sarcopenia: molecular pathophysiology and impact of exercise training. J Cachexia Sarcopenia Muscle 2015;6:197-207.

18. Egerman MA, Glass DJ. Signaling pathways controlling skeletal muscle mass. Crit Rev Biochem Mol Biol 2014;49:59-68.

19. Utech AE, Tadros EM, Hayes TG, Garcia JM. Predicting survival in cancer patients: the role of cachexia and hormonal, nutritional and inflammatory markers. J Cachexia, Sarcopenia and Muscle 2012;3:245-51.

Submission: August, 28, 2019

Revision received: September 27, 2019

Acceptance: September 27, 2019 